\title{
A young male with idiopathic intracranial hypertension with essential thrombocythaemia
}

\author{
Palangasinghe $\mathrm{DR}^{1 *}$, Kularathna $\mathrm{OAR}^{2}$, Herath $\mathrm{HMM}^{3}$ and Pathirana $\mathrm{KD}^{4}$ \\ ${ }^{1}$ Senior Registrar in Medicine, University Medical Unit, Teaching Hospital Karapitiya, Galle, Sri Lanka \\ ${ }^{2}$ Registrar in Medicine, University Medical Unit, Teaching Hospital Karapitiya, Galle, Sri Lanka \\ ${ }^{3}$ Senior Lecturer in Medicine, Consultant Physician, Faculty of Medicine, University of Ruhuna, Sri Lanka \\ ${ }^{4}$ Professor in Medicine, Consultant Neurologist, Faculty of Medicine, University of Ruhuna, Sri Lanka
}

\begin{abstract}
Background: Idiopathic intracranial hypertension (IIH) is a disorder characterized by increased intracranial pressure, normal cerebrospinal fluid (CSF) constituents, without a demonstrable cause for high intracranial pressure. Although commonly associated with obesity, medications like vitamin A, tetracycline and nalidixic acid, it is rarely associated with myeloproliferative disorders. We report a young male from Sri Lanka who developed idiopathic intracranial hypertension associated with essential thrombocythaemia (ET) both which improved with treatment of the latter.

Case presentation: A 24-year-old male presented with persistent frontal headache for two months with transient visual disturbances. On examination he was averagely built, had bilateral optic disc oedema with no ophthalmoplegia, reduced visual acuity or visual field defects. Neuroimaging did not reveal any structural abnormality or venous sinus thrombosis. CSF analysis revealed normal constituents with elevated CSF pressure. Full blood count revealed markedly elevated platelet count $\left(997 \times 10^{3} / \mathrm{mm}^{3}\right)$ with normal other cell lines. Janus kinase (JAK)-2 mutation was positive and bone marrow biopsy was in keeping with ET. He was commenced on cytoreductive therapy for ET and aspirin which resulted in resolution of headache and improvement of platelet count to normal values.
\end{abstract}

Conclusion: IIH could rarely be associated with myeloproliferative disorders like ET. It is important to treat underlying ET which will result in improvement of symptoms secondary to IIH.

\section{Background}

Idiopathic intracranial hypertension (IIH) is a disorder which consists of symptoms and signs of increased intracranial pressure, normal cerebrospinal fluid constituents, without a demonstrable cause for high intracranial pressure. Usually they present with headache and visual symptoms. Headache is the most frequent and the starting symptom in more than $80 \%$ of patients [1]. While bilateral papilloedema is the most characteristic finding some may develop visual field defects and lateral rectus palsy as well. IIH is associated with many different conditions including medications (Vitamin A, Tetracycline, Nalidixic acid, Nitrofurantoin, NSAIDs), systemic arterial hypertension, polycystic ovarian disease, obesity, pregnancy and menstrual irregularities [1]. However, association of IIH with myeloproliferative disorders is very rare. In the literature, only few cases of idiopathic intracranial hypertension were found to be associated with chronic myeloid leukaemia and essential thrombocythaemia [2,3]. We report a case of idiopathic intracranial hypertension associated with essential thrombocythaemia (ET) in a Sri Lankan young male.

\section{Case Presentation}

A 24-year old previously healthy Sri Lankan male presented with a history of moderate to severe persistent headache, predominantly over frontal region for two months. It was not precipitated with coughing, sneezing or straining. It was associated with transient visual obscurations. He denied any history of fever, autonomic symptoms with headache, any altered behaviour or history of head injury. $\mathrm{He}$ had no past history of recurrent headaches. He was a non-smoker and denied any substance abuse including alcohol. He was not on any longterm medications.

On clinical examination, he was found to have bilateral disc oedema with absent retinal venous pulsation (Figure 1). But there was no opthalmoplegia, abnormal pupillary light reflex or visual field defects. Visual acuity was $6 / 6$ in both sides. No other focal neurological signs were detected. Blood pressure was $130 / 80 \mathrm{mmHg}$. Rest of the clinical examination was unremarkable.

Full blood count showed Haemoglobin-13.4g/dL WBC 8740 $\mathrm{mm}^{-3}$. with normal differential count, Platelets - $997 \times 103 / \mathrm{mm}^{-3}$. Magnetic resonance imaging brain (MRI) was normal. Magnetic resonance venogram (MRV) revealed no venous sinus thrombosis. Cerebrospinal fluid manometry revealed increased cerebrospinal fluid pressure $\left(229 \mathrm{~cm} \mathrm{H}_{2} \mathrm{O}\right)$, but normal cerebrospinal fluid constituents (Neutrophils $-0 / \mathrm{mm}^{3}$, Lymphocytes $-1 / \mathrm{mm}^{3}$, Red blood cells -4 / $\mathrm{mm}^{3}$, Protein $-45 \mathrm{~g} / \mathrm{dL}$, Glucose $-86 \mathrm{mg} / \mathrm{dL}$, with random blood glucose of $-128 \mathrm{mg} / \mathrm{dL}$ ). He did not have any evidence of an infection,

Correspondence to: Dhammika Randula Palangasinghe, Senior Registrar in Medicine, University Medical Unit, Teaching Hospital Karapitiya, Sri Lanka, Tel: +94-778-513-896, E-mail: dhammika27@yahoo.com

Key words: Idiopathic intracranial hypertension, essential thrombocythaemia, young male

Received: March 11, 2018; Accepted: March 26, 2018; Published: March 31, 2018 

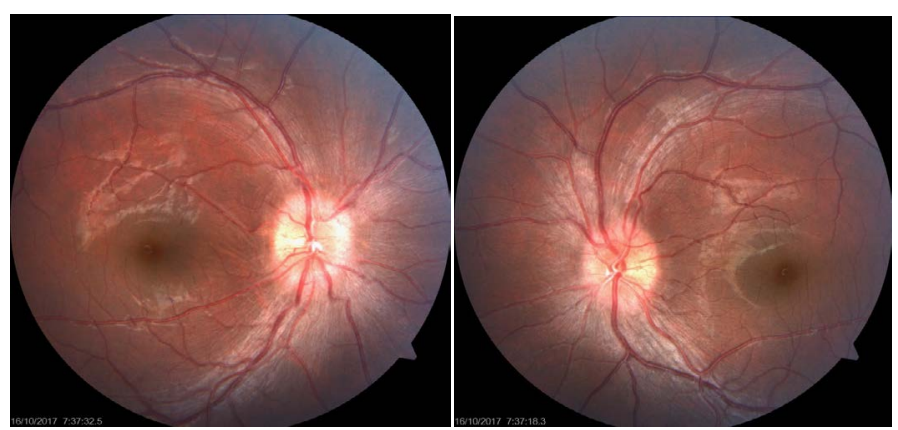

Figure 1. Optic fundi showing bilateral disc edema.

inflammatory disorder or evidence of chronic bleeding to account for a secondary thrombocytosis. Other laboratory investigations were as follows. ESR $-10 \mathrm{~mm}, \mathrm{CRP}-<5 \mathrm{mg} / \mathrm{L}$, serum creatinine -80 $\mu \mathrm{mol} / \mathrm{L}$, alanine transaminase $-47 \mathrm{U} / \mathrm{L}$, aspartate transaminase -27 $\mathrm{U} / \mathrm{L}$, prothrombin time international normalized ratio -1 , activated parital thromboplastin time (APTT) - $28 \mathrm{~S}$, ultrasound scan (USS) of abdomen - did not reveal any splenomegaly. JAK - 2 mutation was detected and findings of bone marrow aspiration and biopsy was in keeping with essential thrombocythaemia. Bone marrow showed normocellular marrow spaces, normal granulopoiesis and erythropoiesis and increased megakaryocytes in number with varying sizes including megakaryocytes with multiple nuclear lobations. But there were no abnormal cell infiltrations.

Patient was initially started on treatment for benign intracranial hypertension with oral acetazolamide $500 \mathrm{mg}$ twice a day. Despite treatment for two weeks, his symptoms and thrombocytosis persisted. Once the diagnosis of ET was confirmed with the availability of JAK-2 mutation report and the bone marrow biopsy findings he was commenced on cytoreductive therapy including oral allopurinol 100 $\mathrm{mg}$ twice daily, oral hydroxyurea $500 \mathrm{mg}$ daily and aspirin $75 \mathrm{mg}$ daily. Review after two weeks of therapy for ET resulted in a significant clinical improvement with full resolution of headache. One month into treatment there was resolution of thrombocythaemia. (Platelet count at one month was $420 \times 103 / \mathrm{mm}^{3}$ ).

\section{Discussion}

Our patient presented with headache and papilloedema with elevated CSF pressure without demonstrable structural abnormality in brain and normal CSF constituents. He also had marked thrombocytosis without any inflammatory disorder, chronic infection or chronic bleeding with positive JAK-2 mutation and evidence of megakaryocyte proliferation in bone marrow. He fulfilled the diagnosis of both IIH and ET. Even though the association of IIH with a myeloproliferative disorder is rare; ET is well known to cause cerebral venous sinus thrombosis [2]. MRI combined with MRV has high sensitivity (97\%) and specificity (99\%) in detection of dural venous sinus thrombosis [5]. In the absence of venous sinus thrombosis in venogram, there are postulated mechanisms with prothombotic conditions to develop high intracranial pressure. One hypothesis is elevated platelet count giving rise to intermittent dural sinus blockage [3]. Other possibility is that unrecognized non-occlusive venous sinus thrombosis from MRV that might occlude the CSF drainage [6]. In the literature mixed prospectively and retrospectively investigated cohort studies showed that many prothrombotic conditions other than thrombocytosis are associated with IIH such as the presence of anticardiolipin antibodies, familial deficiency of anti-thrombin iii, polycythemia vera and increased fibrinogen level [6]. Therefore, looking for an associated such condition is of importance in evaluating a patient with IIH. Our patient's clinical improvement was evident with the resolution of headache with the treatment for two weeks followed by resolution of thrombocythaemia after one month of treatment for ET. This case adds to the literature of association between the IIH and ET. At the same time, it shows the importance of searching for an underlying myeloproliferative disorder in a patient with IIH.

\section{Consent}

Written informed consent was obtained from the patient for the publication of this case and accompanying images.

\section{References}

1. Ball AK, Clarke CE (2006) Idiopathic intracranial hypertension. Lancet Neurol 5: 43342. [Crossref]

2. Pavithran K, Thomas M (2002) Idiopathic intracranial hypertension as the initial manifestation of chronic myeloid leukemia : letter to editor. Neurol India 5: 230. [Crossref]

3. Esack A, Thompson G, Burmester H (1989) Benign intracranial hypertension and essential thrombocythaemia. J NeurolNeurosurg Psychiatry 52: 914. [Crossref]

4. Tehindrazanarivelo A, Bousser MG (1990) Possible benign intracranial hypertension and essential thrombocythaemia. J Neurol Neurosurg Psychiatry 53:819. [Crossref]

5. Chiewvit P, Piyapittayanan S, Poungvarin N (2011) Cerebral venous thrombosis: diagnosis dilemma. Neurol Int 3: e13. [Crossref]

6. Sussman J, Leach M, Greaves M, Malia R, Davies-Jones GA (1997) Potentially prothrombotic abnormalities of coagulation in benign intracranial hypertension. $J$ Neurol Neurosurg Psychiatry 62: 229-233. [Crossref]

7. Williams MW, Vishwanath M (2011) Intracranial hypertension: was it really idiopathic? BMJ Case Rep 2011. [Crossref]

Copyright: (C)2018 Palangasinghe DR. This is an open-access article distributed under the terms of the Creative Commons Attribution License, which permits unrestricted use, distribution, and reproduction in any medium, provided the original author and source are credited. 